\title{
Single Point Interface for Data Analysis in Wireless Sensor Networks
}

\author{
Disha J. Shah \\ PhD Scholar, PACIFIC University \\ Assistant Professor, GLSICA, Ahmedabad, Gujarat, \\ India
}

\begin{abstract}
Today, across a wide variety of areas, huge datasets are being collected and accumulated at a very high pace. The datasets addressed by individual applications are very often heterogeneous and geographically distributed, and are used by the communities of 2users, which are often large and also geographically scattered. Major challenges are involved in the efficient and reliable storage, fast processing, cleaning and extracting descriptive and predictive knowledge from this great mass of data.

In this paper, we describe architecture of single point interface for data analysis meant for different applications of wireless sensor networks. This architecture will help the user extract the exact data required and view the output that is actually needed by him/her. The paper is divided into five sections, section I introduces wireless sensor network, section II lists various applications of WSN, section III contains the sample data set of different applications, section IV gives the proposed solution for performing data analysis and proposed architecture followed by conclusion in section $\mathrm{V}$.
\end{abstract}

\section{General Terms}

Interface, Architecture.

\section{Keywords}

WSN (Wireless Sensor Network), Data Mining, XML (Extensible Markup Language), Data Warehouse, Dataset.

\section{INTRODUCTION}

Wireless sensors are devices that measure a physical quantity and can transmit this information wirelessly to another location. Wireless sensor network (WSN) typically consist of a collection of sensors with their own power supply, wireless communication, data storage, and data processing capability. Using communication between sensor nodes, data from any node can be channeled back to the gateway node and then to the required destination using a local network or Internet. The sensors within a network can be arranged in any topology viz. star, mesh or hybrid [6, 7]. Networks of such embedded devices that work together to provide enhanced monitoring across spatial and temporal scale are growing in popularity [2]. Huge amount of datasets are being gathered due to increase of such networks. Though the data is useful many a times it is not used to its proper capacity.

To use the accumulated data to the proper capacity, data mining is being performed on them. Data mining is the extraction of implicit, previously unknown, and potentially useful information from such data. Various tools and techniques are available for applying data mining in an application [11].

\author{
Harshal A. Arolkar, PhD. \\ Associate Professor, GLSICT, Ahmedabad, \\ Gujarat, \\ India
}

\section{APPLICATIONS OF WSN}

A sensor node can be produced and deployed in large numbers and thus their resources in terms of energy, memory and computational speed and bandwidth can be used. There are different sensors such as pressure, accelerometer, camera, thermal, microphone, etc. They monitor conditions at different locations, such as temperature, humidity, vehicular movement, lightning condition, pressure, soil makeup, noise levels, the presence or absence of certain kinds of objects, mechanical stress levels on attached objects, the current characteristics such as speed, direction and size of an object [10]. Normally these sensor nodes consist of three components: sensing, processing and communicating. WSN Applications are widely used in the domains of Military, Monitoring and Intelligent Transportations [8]. Some examples of application that exist or an evolving are discussed below.

\subsection{Military Application}

WSN is used exclusively in the Military domain. The sensors are usually deployed on the battle field, and sense the unauthorized vehicles and/or intruders and pass this information to its base camp. The base camp then takes appropriate action. Such applications are mission critical and security aspects become major concern in them. [16]

\subsection{Monitoring applications of WSN}

WSN is being used in various specialized monitoring application areas such as forest fire monitoring, medical care, and agriculture monitoring, structural monitoring. A brief discussion of these applications is as mentioned.

\subsection{Forest Fire Monitoring}

Sensors are deployed in the forest which will detect and predict forest fire on time and accurately in order to minimize the loss of forests, wild animals and people in the forest. The sensors will collect data and send it to respective cluster nodes that collaboratively process the data and take appropriate action [15]. Many forest fire surveillance systems have also been proposed [3]

\subsection{Medical Care}

Sensor networks are also widely used in health care area. In some modern hospitals, sensor networks are constructed to monitor patient physiological data, to control the drug administration, track and monitor patients and doctors who are inside a hospital. The motion sensors are used to monitor the user's overall activity and an ECG sensor is used for monitoring heart activity [5]. Many health care monitoring systems have been implemented till now [1] 


\subsection{Agricultural Monitoring}

It is very important to monitor a crop growth and field environmental information in various places under harsh conditions over long periods of time [18].A system is proposed to monitor the growing process and can also control the environment of green house [17]. This results in improvement of product quality.

\subsection{Structural Monitoring}

Structural Health Monitoring is one of the important domains of WSN. Structures like heavy duty bridges, tall buildings, etc. should be inspected at regular time intervals, and repairing or replacing based on the time of use, rather than on their working conditions [19].

\subsection{Habitat Monitoring}

Habitat monitoring enables researchers worldwide to engage in non-intrusive and non-disruptive monitoring of sensitive wildlife and habitats [13]. The GPS enabled sensor nodes can be incorporated in collars of animals and geographical location data was passed to abase-station using, where necessary, other network nodes as repeaters [14].

\section{SAMPLE DATA SET}

As seen in previous section there are different applications of WSN. Each application would gather data frequently in some specified but unique format. The analysis of each data set is tedious and cumbersome task. Following are the few sample data sets collected from different set of applications.

\subsection{Weather Monitoring}

In the past few years, the occurrences of natural disasters have been continuously changing our lives, damaging property and lifestyle in many different ways. A sensor network is used which will send the weather information and can be used to prevent enormous damage from natural calamities [4].

Table 1. Weather Data of Davis Weather Station in Amherst, Massachusetts

\begin{tabular}{|c|c|c|c|c|c|c|c|c|c|c|c|c|c|}
\hline Timestamp & Temp & Chill & HIndex & Humid & Dewpt & Wind & HiWind & WindDir & Rain & Barom & Solar & ET & $\mathbf{U V}$ \\
\hline 20060214 00:05 & 26.4 & 21.6 & 26.4 & 62 & 15.2 & 4 & 10 & 202 & 0 & 29.959 & 0 & 0 & 0 \\
\hline $2006021400: 15$ & 26.6 & 26.6 & 26.6 & 62 & 15.4 & 2 & 6 & 180 & 0 & 29.959 & 0 & 0 & 0 \\
\hline $2006021400: 25$ & 26.6 & 26.6 & 26.6 & 62 & 15.4 & 3 & 6 & 202 & 0 & 29.959 & 0 & 0 & 0 \\
\hline $2006021400: 35$ & 26.6 & 26.6 & 26.6 & 63 & 15.7 & 3 & 6 & 180 & 0 & 29.961 & 0 & 0 & 0 \\
\hline $2006021400: 45$ & 26.9 & 26.9 & 26.9 & 62 & 15.6 & 3 & 8 & 180 & 0 & 29.963 & 0 & 0 & 0 \\
\hline $2006021400: 55$ & 26.7 & 26.7 & 26.7 & 63 & 15.8 & 2 & 6 & 202 & 0 & 29.963 & 0 & 0 & 0 \\
\hline $2006021401: 05$ & 26.7 & 22 & 26.7 & 64 & 16.2 & 4 & 9 & 202 & 0 & 29.965 & 0 & 0 & 0 \\
\hline
\end{tabular}

In Table 1, the data was collected every 5 minutes over a 15 month period from Feb 2006 to May 2007. We have shown here the data at 10 minutes interval. The weather station contained sensors for measuring temperature, wind chill, humidity, dew-point, wind, wind direction, rain, pressure, sunlight, and UV.

\subsection{Cattle Monitoring}

Table 2. Cattle Movement \& Behavior Monitoring in University of Nottingham's Dairy Centre

\begin{tabular}{|c|c|c|c|c|c|c|c|c|}
\hline Type & Time & Date & Lat & Lon & Timeoffix & Status & SOG & COG \\
\hline GPS & 1152012609000 & 040706 & 52.839488 & -1.251200 & 103111.879 & $\mathrm{~V}$ & 0.0 & 0.0 \\
\hline GPS & 1152012618000 & 040706 & 52.839488 & -1.251200 & 103120.878 & $\mathrm{~V}$ & 0.0 & 0.0 \\
\hline GPS & 1152012628000 & 040706 & 52.839488 & -1.251200 & 103129.878 & $\mathrm{~V}$ & 0.0 & 0.0 \\
\hline GPS & 1152012637000 & 040706 & 52.839488 & -1.251200 & 103138.877 & $\mathrm{~V}$ & 0.0 & 0.0 \\
\hline GPS & 1152012646000 & 040706 & 52.839488 & -1.251200 & 103148.877 & $\mathrm{~V}$ & 0.0 & 0.0 \\
\hline GPS & 1152012655000 & 040706 & 52.839488 & -1.251200 & 103157.876 & $\mathrm{~V}$ & 0.0 & 0.0 \\
\hline GPS & 1152012664000 & 040706 & 52.839488 & -1.251200 & 103206.876 & $\mathrm{~V}$ & 0.0 & 0.0 \\
\hline GPS & 1152012673000 & 040706 & 52.839488 & -1.251200 & 103215.875 & $\mathrm{~V}$ & 0.0 & 0.0 \\
\hline GPS & 1152012680000 & 040706 & 52.839488 & -1.251200 & 103221.875 & $\mathrm{~V}$ & 0.0 & 0.0 \\
\hline
\end{tabular}


Table 2 contains traces of data collected every 3 minutes from 04 July 2007 to 13 July 2007 . We have shown here at the interval of 9 minutes. The sensors measured, date of the measurement, latitude, longitude, time in GMT, status, course of the ground, mode, number of satellites used for the fix, position dilution of precision, horizontal dilution of precision and vertical dilution of precision.

There are numerous applications of wireless sensor networks. Each field/application gathers some data, analysis the data and takes corrective actions based on the analysis done. In each application, it has its own interface which does the aggregation and analysis of data.

Here, we are proposing single point architecture for data analysis of data collected through different applications in wireless sensor networks. With the help of the interface, the user would be able to analyze the data, irrelevant of the application he/she is using to gather it.

\section{PROPOSED SOLUTION \& ARCHITECTURE}

In this paper, we propose a single point interface for data analysis of data gathered using WSN architecture. Using this interface, we intend to analyze the data so that the user can effortlessly do a comparison with the previous analysis done and then can take decisions. The user can also integrate an interface easily in the respective domain.

The proposed solution can be divided into seven different phases. First, the data is to be collected and its pattern is to be

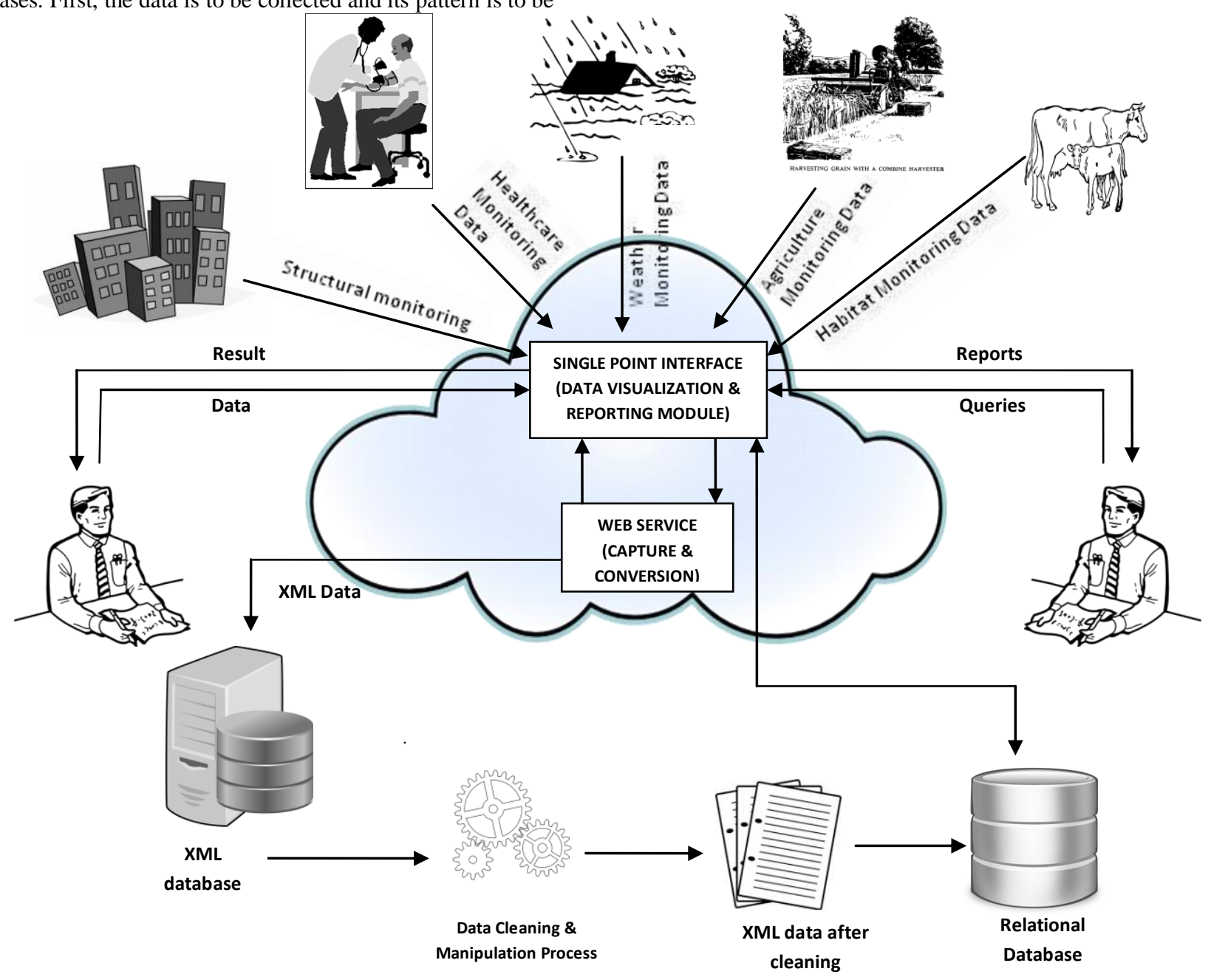

identified from different applications of WSN. Then the data is stored in XML which has self-documenting format that describes structure, field names and its values. The concept of data warehouse is used for data storage. Decision making is a critical aspect in any application; the Decision Support System helps in managerial decision making, especially with unstructured information. The web-service would be created for the support of decision system and it can be used through the single point interface. The web service can be added in any domain and it would be helpful in taking decisive actions.

The architecture shown in the Figure 1, shows that each application will provide the data in their own format to the single point interface. The proposed architecture will provide a web service which would capture the data of the respective application. Once the data is captured, the data is stored in the warehouse. Data warehouse is used for managing large amounts of data in an effective and efficient manner [20]. Once the data has been gathered, the data cleaning and manipulation process is done where the data is being filtered in a standard format as per the requirements of the user. The XML data generated is stored in relational database.

The results of the data analysis can be obtained in different formats as per user's requirements. Like, the user may generate tabular outputs, pie charts if required. The graphical user interface is web-based, and it allows remote interaction. The workflow of the proposed architecture is shown in Figure 2.

Fig 1: Single Point Architec 


\section{CONCLUSION}

With the increase in usage of wireless sensor networks for gathering data, huge amount of data is being gather at rapid pace. Researchers usually spend more time in developing a GUI and analyzing the data. The proposed architecture will provide a single point interface to the user for data analysis irrespective of its application domain.

The main idea here is to allow user to incorporate a graphical interface easily into his respective domain. The GUI will allow the researcher to concentrate on analysis algorithms. It will also provide in depth and detailed information of the data gathered. The user can easily compare the result of the newly gathered data set with the previous analysis and then can take required decisions.
The facility of universal data accessibility will also help the user to perform the operations across remote destinations. As the analysis of the data can be done faster, it would result in getting more time for taking actions. The resultant web services will be a software system designed to support interoperable machine to machine interaction over a network. The web services would be self-contained and self-describing and will communicate using open protocols. Using this web service, the user will be able to exchange data between different applications and different platforms.

We intend to use open source technology for the development of the architecture, so the cost would not be a problem. Thus an overall enhancement would be specified by giving a single point framework for data analysis of data collected using wireless sensor networks.

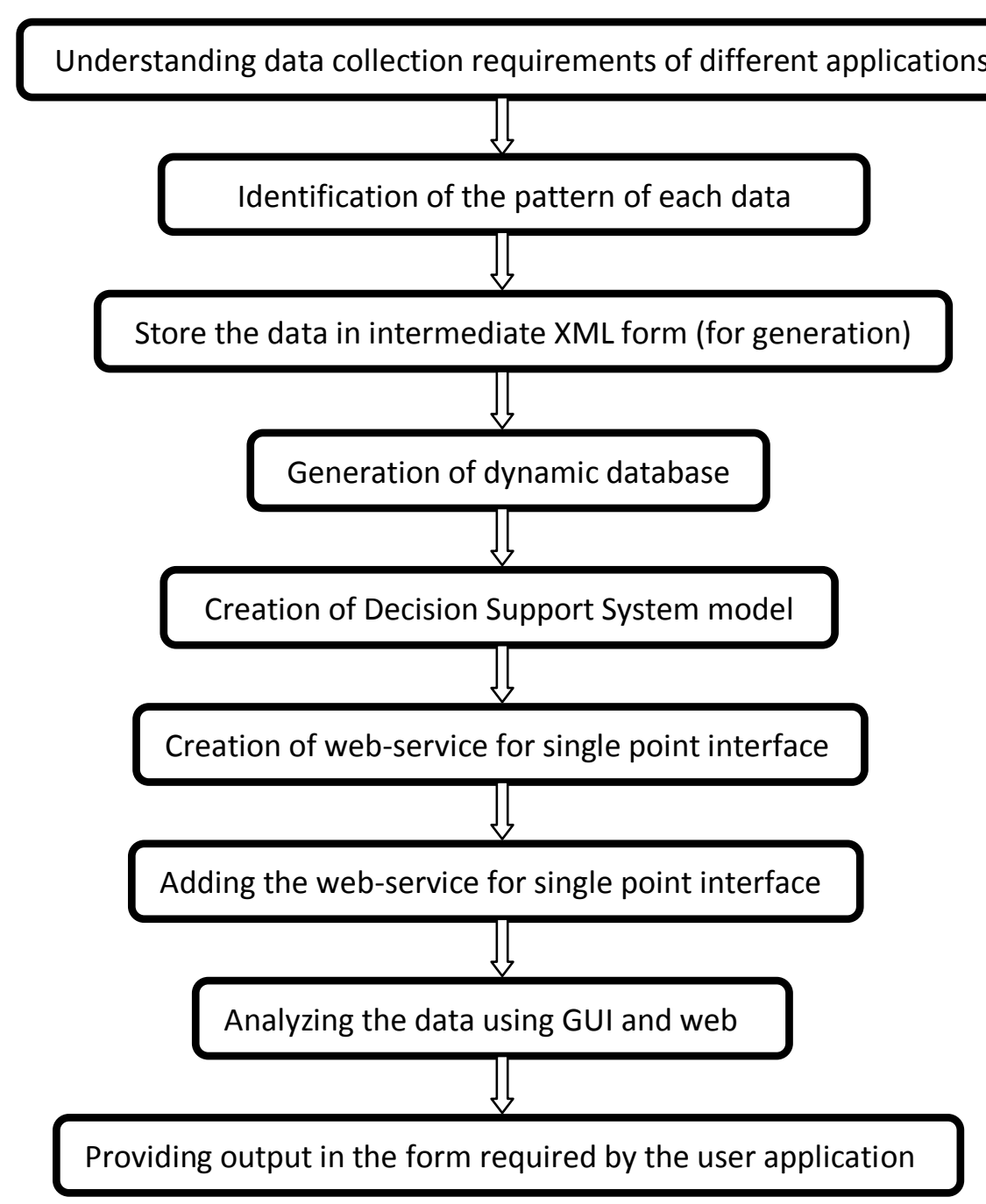

Fig 2: Workflow of the Architecture 


\section{REFERENCES}

[1] Abhiman Hande, Todd Polk, William Walker, Dinesh Bhatia, "Self- Powered Wireless Sensor Networks for Remote Patient Monitoring in Hospitals", Sensors, 2006, Vol. 6, pp. 1102-1117, ISSN 1424-8220..

[2] Bhaskar Krishnamachari, "Networking Wireless Sensors", Cambridge University Press, 2005, ISBN: 0521838479 .

[3] Byungrak Son, Yong-Sork Her, Jung-Gyu Kim, "A Design and Implementation of Forest-Fires Surveillance System based on Wireless Sensor Networks for South Korea mountain", IJCSNS International Journal of Computer Science and Network Security, 2006, Vol. 6, pp. 124-130.

[4] Cholatip Yawut, Sathapath Kilaso, "A Wireless Sensor Network for Weather and Disaster Alarm Systems", 2011 International Conference on Information and Electronics Engineering, 2011, Vol. 6, pp. 155-159.

[5] Chris Otto, Aleksandar Milenkovic, Corey Sanders, Emil Jovanov, "System Architecture of a Wireless Body Area Sensor Network for Ubiquitous Health Monitoring ", Journal of Mobile Multimedia, 2006, Vol. 1, pp. 307326, ISSN: 1918-2104.

[6] Chun-Ta Li, "Security of Wireless Sensor Networks: Current Status and Key Issues", InTech, Smart Wireless Sensor Networks, 2010, pp. 300-313, ISBN 978-953307-261-6.

[7] Cook, D. J. and Das, "Smart Environments: Technologies, Protocols, and Applications", Wiley Publications, 2004, ISBN: 978-0-471-54448-7.

[8] Harshal Arolkar, Kashyap Dhamecha, Darshan Patel, "Architecture for Accident Monitoring in BRTS Corridors Using WSN ", International Journal of Enterprise Computing and Business Systems, 2012, Vol. 2, 1, ISSN: 2230-8849.

[9] Hillol Kargupta, Ilker Hamzaoglu, Brian Stafford, "Scalable, Distributed Data Mining-An Agent Architecture", KDD-97 Proceedings, 1997, pp. 211-214.

[10] I.F. Akyildiz, Weilian Su; Sankarasubramaniam, Y.; Cayirci, E, " Wireless sensor networks: a survey", Communications Magazine, IEEE, 2002, Vol. 40, 8, pp. 102-114, ISSN: 0163-6804.

[11] Ian H. Witten, Eibe Frank, "Data Mining: practical machine learning tools and techniques", Elsevier, 2005, ISBN: 0-12-088407-0.
[12] Kae Hsiang Kwong, Tsung Ta Wu, Hock Guan Goh, Bruce Stephen, Michael Gilroy, Craig Michie, and Ivan Andonovic,"Centre for Intelligent Dynamic Communications, Department of Electronic and Electrical Engineering, University of Strathclyde, Glasgow, United Kingdom ", Wireless Sensor Networks in Agriculture: Cattle Monitoring for Farming Industries, PIERS Online, 2009, Vol. 5, 1, pp. 31-35. ISSN: 19317360.

[13] Kazem Sohraby, Daniel Minoli, Taieb Znati, "Wireless Sensor Networks: Technology, Protocols and Applications", Wiley Publications, 2007, ISBN: 978-0471-74300-2.

[14] Konstantinos Sasloglou, Ian A Glover, Kae-Hsiang Kwong and Ivan Andonovic, " Wireless Sensor Network for Animal Monitoring using both Antenna and Basestation Diversity", 11th IEEE Singapore International Conference on Communication Systems, 2008. ICCS 2008, 2008, pp. 27-33, E-ISBN: 978-1-4244-2424-5.

[15] Liyang Yu, Neng Wang; Xiaoqiao Meng, " Real-time forest fire detection with wireless sensor networks ", Proceedings 2005 International Conference on Wireless Communications, Networking and Mobile Computing, 2005, Vol. 2, 23-26, pp. 1214-1217, ISBN: 0-7803-9335$\mathrm{X}$.

[16] Michael Winkler, Klaus-Dieter Tuchs, Kester Hughes, and Graeme Barclay, "Theoretical and practical aspect of military wireless sensor network ", Journal of Telecommunication and Information Technology, 2008, pp. $37-45$.

[17] Seong-eunYoo, Jae-eon Kim, Taehong Kim, Sungjin Ahn, Jongwoo Sung, Daeyoung Kim, " Automated Agriculture System based on WSN", IEEE International Symposium on Consumer Electronics, 2007, 2007, pp. 15, E-ISBN: 978-1-4244-1110-8.

[18] Tokihiro Fukatsu, Masayuki Hirafuji, "Field Monitoring Using Sensor-Nodes with a Web Server", Journal of Robotics and Mechatronics, 2005, Vol. 17, 2, pp.164175 .

[19] Vivek Katiyar, Narottam Chand, Naveen Chauhan, "Recent advance and future trends in wireless sensor networks", International Journal of applied engineering research, Dindigul, 2010, vol. 1, 3, pp. 330-342, ISSN: ᄀ 0976 $\neg 4259$.

[20] William Inmon, "Building the Data Warehouse", Wiley India Pvt. Ltd, 4th Edition, ISBN: 9788126506453. 